\title{
BEPCII-THE SECOND PHASE CONSTRUCTION OF BEIJING ELECTRON POSITRON COLLIDER
}

\author{
C. Zhang, G.X. Pei for BEPCII Team \\ IHEP, CAS, P.O. Box 918, Beijing 100039, P.R. China
}

\begin{abstract}
BEPCII, the second phase construction of the Beijing Electron Positron Collider (BEPC), is a double ring machine with a design luminosity of $1 \times 10^{33} \mathrm{~cm}^{-2} \mathrm{~s}^{-1}$ at $1.89 \mathrm{GeV}$, two orders of magnitude higher than present BEPC. The construction was started in the beginning of 2004 and is scheduled to be completed by the end of 2007. The upgrading of the collider should also provide an improved SR performance with higher beam energy and intensity. The beam currents will be increased to $250 \mathrm{~mA}$ at $\mathrm{E}=2.5 \mathrm{GeV}$ for the dedicated synchrotron radiation operation. Some key technologies, such as superconducting RF cavity, superconducting micro-beta quadrupole and low impedance vacuum devices etc., are being developed in order to achieve the target of the BEPCII.
\end{abstract}

\section{INTRODUCTION}

The $\mathrm{BEPC}^{[1]}$ has been well operated for more than 15 years with many exciting high energy physics and synchrotron radiation research results since it was put into operation in 1989. As a natural extension, the BEPCII, including an upgraded injector linac and two storage rings replacing the old one in the existing tunnel, has started its construction since the beginning of 2004 with a construction period of 4 years, aiming at a luminosity of $1 \times 10^{33} \mathrm{~cm}^{-2} \mathrm{~s}^{-1}$ at $1.89 \mathrm{GeV}$ (two orders of magnitude higher). As an essential requirement to its injector linac, on energy injection with an injection rate of $50 \mathrm{~mA} / \mathrm{min}$ for the positron beam is necessary for getting a higher average luminosity. Thus a lot of upgrades will be carried out to accomplish the task, for example, a high yield new positron production system. As to the storage rings, the design and construction are extremely difficulty since the existing BEPC tunnel is only 240 meters in circumference, much smaller compared to other high luminosity machines. The luminosity design philosophy is to increase the beam current from $35 \mathrm{~mA}$ to about $900 \mathrm{~mA}$ with multi-bunch, and reduce the Beta-function at interaction point $\beta_{y}{ }^{*}$ from $5 \mathrm{~cm}$ to $1.5 \mathrm{~cm}$. And the design should also provide an improved synchrotron radiation operation with a higher intensity of $250 \mathrm{~mA}$ at $2.5 \mathrm{GeV}$. Thus, numbers of key technologies have to be used, such as superconducting RF cavity, superconducting micro-beta quadrupole, two-inone magnet, bunch-by-bunch feedback and low impedance vacuum devices etc. So far, the BEPCII design has been completed, and almost all systems, especially the key devices are well prepared and ready for installation. According to the schedule, the old machine removal and the new machine installation will soon start from the early July, 2005 and be completed in about 10 months.

\section{THE BEPCII DESIGN}

The BEPCII ${ }^{[2,3]}$ will be operated in the energy region of $1.0-2.1 \mathrm{GeV}$, so that its physical potential in $\tau$ and charm range is well preserved. As the physics is concentrated on the $\mathrm{J} / \psi(3097), \psi(3686), \psi(3770)$ and nearby, the collider is optimized at the energy of $1.89 \mathrm{GeV}$.

\section{Luminosity Issue}

The luminosity for an $e^{+}-e^{-}$collider can be expressed as

$L\left(\mathrm{~cm}^{-2} \mathrm{~s}^{-1}\right)=2.17 \times 10^{34}(1+r) \xi_{y} \frac{E(G e V) k_{b} I_{b}(\mathrm{~A})}{\beta_{y}^{*}(\mathrm{~cm})}$,

where $r=\sigma_{y}^{*} / \sigma_{x}^{*}$ is the beam aspect ratio at the interaction point (IP), $\xi_{y}$ the vertical beam-beam parameter, $\beta_{y}{ }^{*}$ the vertical envelope function at IP, $k_{b}$ the bunch number in each beam and $I_{b}$ the bunch current. From the formula, the design strategy to enhance the luminosity is to adopt micro- $\beta$ technique and to increase the bunch number with double ring scheme. Figure 1 is the geometry layout of the BEPCII storage rings.

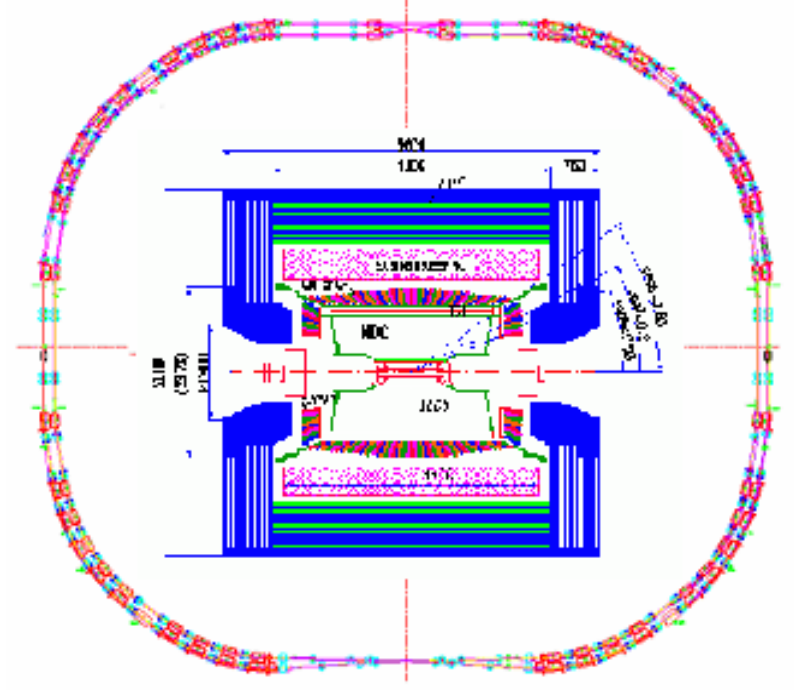

Figure 1: The layout of the double ring of the BEPCII.

The electron ring and the positron ring cross each other in the northern and the southern IP's. The horizontal crossing angle between two beams at the southern IP, where the detector located, is $11 \mathrm{mrad} \times 2$ to make enough separation, but cause no significant degradation to the 
luminosity. While in the northern IP, the two beams cross horizontally with an angle of $154.7 \mathrm{mrad} \times 2$ and a vertical bump is used to separate two beams, so that the optics of the two rings can be symmetric. For the dedicated synchrotron radiation operation mode, the electron beam must circulate in the outer ring. So a bypass bridges the two outer half rings in the northern Interaction Region (IR), and in the southern IR, a pair of bending coils in superconducting magnet assembly serves this purpose.

\section{Beam Physics}

Based on the strategy of the luminosity upgrading of the BEPC, the design for the BEPCII has been worked out. The beam physics issues are intensively studied on the aspects of lattice design, beam-beam interaction, and collective effects etc. [4].

The lattice for the collision mode is optimized to improve the dynamic aperture against various errors including misalignment and multipole field of magnets, meantime, with consideration on tuning the beta function to reduce the background due to Touschek effect. The lattice for SR mode is optimized on low emittance. Table 1 summarizes the main parameters of the BEPCII machine.

Table 1: Main parameters of the BEPCII storage ring.

\begin{tabular}{|c|c|c|c|}
\hline Parameters & Unit & Collision & SR \\
\hline Energy & $\mathrm{GeV}$ & 1.89 & 2.5 \\
\hline Circumference & $\mathrm{m}$ & 237.53 & 241.13 \\
\hline RF frequency & $\mathrm{MHz}$ & 499.8 & 499.8 \\
\hline Harmonic & & 396 & 402 \\
\hline RF voltage & MV & 1.5 & 3.0 \\
\hline Transverse tunes & & $\begin{array}{l}6.53 / 7.58 \\
6.53 / 5.58\end{array}$ & $8.28 / 5.18$ \\
\hline Damping time & $\mathrm{ms}$ & $25 / 25 / 12.5$ & $12 / 12 / 6$ \\
\hline Beam current & A & 0.91 & 0.25 \\
\hline Bunch number & & 93 & Multi \\
\hline SR loss per turn & $\mathrm{keV}$ & 121 & 336 \\
\hline SR power & $\mathrm{kW}$ & 110 & 84 \\
\hline Energy spread & & $5.16 \times 10^{-4}$ & $6.66 \times 10^{-4}$ \\
\hline Compact factor & & 0.0235 & 0.016 \\
\hline Bunch length & $\mathrm{cm}$ & 1.5 & 1.18 \\
\hline Emittance & $\mathrm{nm} \cdot \mathrm{rad}$ & $144 / 2.2$ & $120 /$ \\
\hline$\beta$ function at IP & $\mathrm{m}$ & $1 / 0.015$ & - \\
\hline Crossing angle & mrad & $11 \times 2$ & - \\
\hline Bunch spacing & $\mathrm{m}$ & 2.4 & - \\
\hline $\begin{array}{l}\text { Beam-beam } \\
\text { Parameter }\end{array}$ & & $0.04 / 0.04$ & - \\
\hline Luminosity & $\mathrm{cm}^{-2} \mathrm{~s}^{-1}$ & $1.0 \times 10^{33}$ & - \\
\hline Luminosity & $\mathrm{cm}^{-2} \mathrm{~s}^{-1}$ & $1.0 \times 10^{33}$ & - \\
\hline
\end{tabular}

Beam-beam simulation is done to choose the best working point for high luminosity. It is found that when the fraction parts of the horizontal and vertical tunes are around $0.53 / 0.58$, respectively, the luminosity is the highest. Simulation also shows that the crossing angle of $11 \mathrm{mrad} \times 2$ is acceptable and beam-beam parameter of 0.04 is reasonable and reachable.
The single beam collective effects in the BEPCII include the single and multi-bunch instabilities due to impedance as well as the ion effect in the electron ring and the electron cloud (ECI) in the positron ring. Our main concern is the impedance control to alleviate bunch lengthening and to control ECI. A strict impedance budget is set and antechamber with TiN coating is adopted against ECI. The detailed studies can refer to [5].

The beam lifetime is around 3 hours, with the main limits coming from the beam-beam bremsstrahlung, Touschek effect and beam-gas interaction. With top-off injection, the maximum average luminosity can reach more than $60 \%$ of the peak luminosity.

\section{KEY TECHLOLOGIES \& SYSTEMS}

Many key technologies and hardware subsystems have been developed for the BEPCII. Some are challenging because they are new to us, such as superconducting RF cavity, superconducting magnet, cryogenics, bunch-bybunch feedback etc., some are hard because of the space limited, such as vacuum system, interaction region (IR) etc.

\section{Injector Linac $^{[6]}$}

The BEPC injector is a 202meter electron linac with 16 $\mathrm{RF}$ power sources and $56 \mathrm{~S}$-band RF structures. The positron source is located at down stream of the second RF power station.

For the energy upgrade from $1.3 \mathrm{GeV}$ to $1.89 \mathrm{GeV}$ for the top-off injection, $50 \mathrm{MW}$ klystrons will be used to replace the present $30 \mathrm{MW}$ ones. The klystron modulators will be rebuilt for higher beam voltage of $320 \mathrm{kV}$ and $50 \mathrm{~Hz}$ operation. The required positron injection rate of $50 \mathrm{~mA} / \mathrm{min}$ is a challenging job, which is almost 20 times as much as the BEPC number, and a lot of measures are taken as listed in Table 2. So we need a new electron gun, a new positron source with a flux concentrator, a new RF power system with its phasing loop and a beam tuning system with the orbit correction and optics tuning etc. Figure 2 is the picture of the BPECII positron source, which was installed into the linac tunnel last summer.

Table 2: Technical measures for increasing $\mathrm{e}^{+}$intensity.

\begin{tabular}{|l|c|}
\hline \multicolumn{1}{|c|}{ Technical measure } & Gain \\
\hline $\begin{array}{l}\text { To increase } \mathrm{e}^{-} \text {beam current on } \mathrm{e}^{+} \text {target } \\
I_{G}=2.5 \mathrm{~A} \nearrow\end{array}$ & 2.4 \\
\hline $\begin{array}{l}\text { To increase the repetition rate } \\
f_{r}=12.5 \mathrm{~Hz} \pi 50 \mathrm{~Hz}\end{array}$ & 4 \\
\hline $\begin{array}{l}\text { To enhance bombarding energy for } \mathrm{e}^{+} \\
E_{c}=140 \mathrm{MeV} \pi 240 \mathrm{MeV}\end{array}$ & 1.7 \\
\hline $\begin{array}{l}\text { New positron source of higher yield } \\
\eta=1.4 \% \pi 2.7 \%\left(\mathrm{e}^{+} / \mathrm{e}^{-} \cdot \mathrm{GeV}\right)\end{array}$ & 1.9 \\
\hline Two bunches injection $n_{b}=1 \nearrow 2$ & 1.6 \\
\hline Reduction of pulse length $T=2.5 \mathrm{~ns} У 1 \mathrm{~ns}$ & 0.4 \\
\hline Total intensity enhancement factor & 19.8 \\
\hline
\end{tabular}




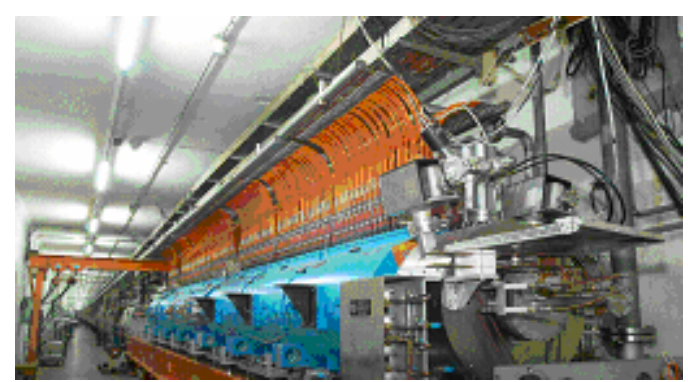

Figure 2: The Picture of BEPCII positron source.

\section{$500 \mathrm{MHz}$ Supercunducting RF System}

As mentioned in the previous sections, a short beam bunch of $1.5 \mathrm{~cm}$ is very important to realize the high luminosity goal, and the proper way for shortening the bunch length is to increase the RF frequency and enhance the accelerating voltage in the cavity.

We have chosen the superconducting cavity for its high accelerating gradient, smooth structure and large beam port, well damped HOMs and low RF power consuming. There will be two $500 \mathrm{MHz}$ superconducting cavities for the BEPCII two rings, each will be powered with a 250 $\mathrm{kW}$ klystron and provide 1.5MV RF voltage.

For the cryogenic system, two $500 \mathrm{~W}$ refrigerators will be applied to the BEPCII. One is for the superconducting RF cavities and the other is for the superconducting insertion magnets and the detector solenoids in the IR.

\section{Injection Kickers}

The BEPCII needs two injection systems for both electron and positron beam filling, and each consists of two fast kickers and one Lambertson magnet. The kicker should provide an integrated field of 200 Gauss-meter in a 600 ns half sinusoidal waveform with a field uniformity of better than $\pm 1 \%$. Experience from the BEPC and the other machines showed that the kicker magnet could be one of the major contributors to the coupling impedance. And attention should be paid to the BEPCII injection kicker design.

We adopted a modified slotted pipe kicker design with the coating strips on ceramic bar instead of metallic plates as the beam image current return paths to improve the field efficiency. The OPERA and MAFIA simulations showed a $\pm 0.6 \%$ field uniformity at $\mathrm{y}=0$ plane and a coupling impedance of less than $0.022 \Omega$.

\section{Magnets and Power Supplies}

Totally, there are 357 magnets for the two rings, including 44 old and 48 new bends, 28 old and 89 new quads, 72 new sextuples, 22 old and 54 new dipole correctors. Here, old means the magnets reused from BEPC. Due to space limitation of the existing BEPC tunnel, the inner ring magnets should be as small as possible. The magnet design should also consider giving enough room for the antechamber on horizontal plane. Forty girders in the arcs of each ring are used to mount these magnet assembles as shown in Figure 3. All these magnets are powered by 326 DC power supplies. And two types of DC power supplies, i.e. switch-mode (Chopper, pulse width modulation converter and zero voltage switching converters) and thyristor phasecontrolled type (12 and 6 phases rectifiers), are applied to the BEPCII storage rings.

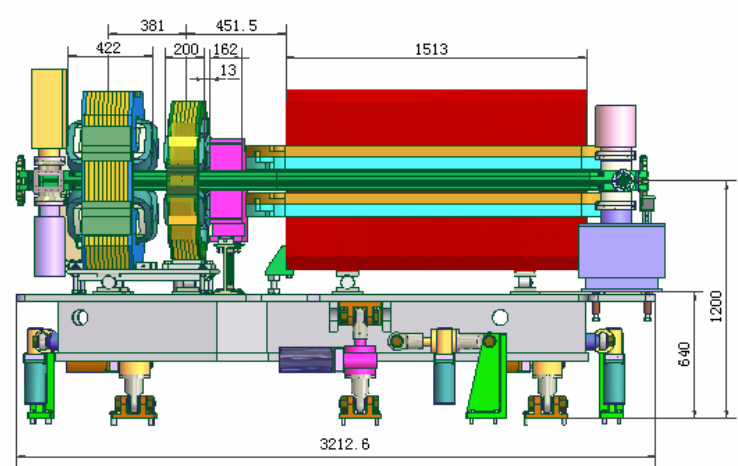

Figure 3: The magnets mounted on a girder.

\section{Vacuum System}

The BEPCII poses two challenges to the vacuum system, one is the vacuum pressure, and the other is the impedance.

The dynamic vacuum at a high beam current should satisfy the requirements of the sufficient beam lifetime, and the low background in the IR. The design vacuum pressure of the machine is $8 \times 10^{-9}$ Torr in the arc and $5 \times 10^{-10}$ Torr in the IR. The distributed ion pumps are used inside the bending sections, while in the straight sections lumped ion pumps and NEG are applied.

To reduce impedance, the vacuum chamber should be as smooth as possible. Antechambers will be used for both electron and positron rings. And for the positron ring, concerning the ECI, the inner surface of the beam pipe in the arc should be coated with TiN to reduce the secondary electron yield (SEY). Some masks are placed upstream to such vacuum components as bellows in order to prevent them from exposure to synchrotron radiation.

\section{IR and Superconducting Insertion Magnets}

The design of IR has to accommodate competing and conflicting requirements from the accelerator and the detector. Many kinds of equipments, including magnets, beam diagnostic instruments, masks, vacuum pumps, and experiment detector, must coexist in a very small region. Figure 4 demonstrates a 3D sketch of the half IR. 


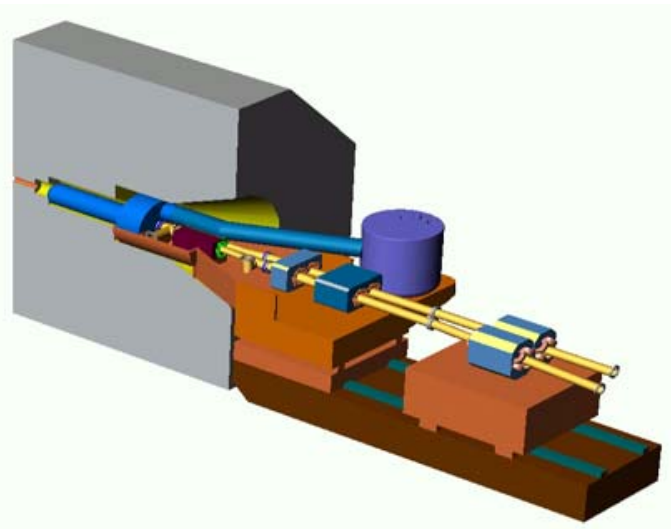

Figure 4: A 3D sketch of the IR.

The support, installation, background shielding, vacuum pumping and many other issues in the IR are carefully studied. A mock-up of IR installation is carried out to demonstrate the designed procedure.

A special pair of superconducting IR magnet assembly are designed with main and skew quadrupole, compensation solenoid and dipole coils to squeeze the $\beta$ function at IP, compensating the detector solenoid and serve as the bridge connecting outer rings in SR operation, respectively.

The superconducting magnet is designed and made collaborating with BNL.

Some special warm bore magnet in IR as septum bending magnet and two in one quadrupoles have been manufactured, the magnetic field measurement results confirms the design

\section{Instrumentation and Control}

The instrumentation system provides precise and sufficient information about the beams, particularly the ability to finely tune the beam orbit to achieve exact collision for both beams as well as the high luminosity. It consists of 132 beam position monitors (BPM's), 2 DCCT's, 2 bunch current monitors, 8 fluorescent screen monitors and 2 SR monitors in the BEPCII storage rings. Since the growth time of the most dangerous transverse coupled bunch instabilities can reach the level of $2 \mathrm{~ms}$, a fast bunch-by-bunch transverse feedback system will be equipped in order to damp the beam instabilities.

The control system, based on the EPICS environment to provide a friendly man-machine interface for operators, contains the computer control, timing, interlock and the communication subsystems. Attention has been paid to the time jitter for stable injection, and the storage ring frequency and the injector linac frequency must be phaselocked.

\section{SCHEDULE AND PROGRESS}

The BEPCII project was proposed many years ago, and the single ring "pretzel" design was in principle approved in July of 2000. In an important international review meeting on the feasibility study held on April 2 6, 2001, the review committee strongly recommended the double ring design for its lower risk in attaining the design luminosity. Since then, we submitted a feasibility study report of the double ring scheme on March 19, 2003, and a preliminary design report on Sept. 15, 2003, and finally got approved on Oct. 15, the same year. After its approval, the project was scheduled to be completed in 4 years. The main mile stones are listed here:

- May 1 to Oct.1, 2004: BESII removal and linac upgrade installation

- Dec.29, 2004 to June 30, 2005: BEPC operation for dedicated synchrotron radiation

- July 1 to 30, 2005: Old ring removal

- Aug. 1 to Sept.30, 2005: Infrastructure upgrade

- Oct.1 to Dec.31, 2005: Ring installation

- Jan.1 to March 31, 2006: Alignment and survey

- April 1 to May 31, 2006: Subsystem tuning

- June 1 to Dec.31, 2006: Ring commissioning without detector and dedicated SR operation

- Jan.1 to Feb.28, 2007: Moving BESIII detector into the interaction point

- March 1 to 31, 2007: Ring commissioning with the BESIII detector

- From April 1 to the end of 2007: Test operation and operation

General speaking, up to now, all systems' technique design, prototype, mass production and testing were going smoothly. For the injector linac upgrade, except for the beam energy because there are still three RF power sources remaining to be rebuilt, a $500 \mathrm{~mA}$ electron beam with $0.14 \mu \mathrm{m}$ beam emittance at $1.3 \mathrm{GeV}$ has been obtained. On March 19, 2005, we got the first positron beam of $84 \mathrm{~mA}$ and $48 \mathrm{~mA}$ at the positron source exit and the linac end, respectively. In a machine study conducted during April 22 to 24 , a stable $60 \mathrm{~mA}, 1.3 \mathrm{GeV}$ positron bunch has been delivered to storage ring. The primary electron beam was $6.5 \mathrm{~A}, 210 \mathrm{MeV}$ with a beam size of about $1.5 \mathrm{~mm}$ in radius $(1 \sigma)$.

As to the storage ring, almost all the magnets and the power supplies were delivered and tested with satisfaction. 30 antechambers delivered to date with typical rate of 10 per month, so total of 80 antechambers can be delivered by the end of October 2005. Figure 5 is the picture of antechamber been manufactured. The antechamber TiN coating test has been finished, and the coating rate could be 7-8 per month with two sets of coating devices. The two superconducting RF cavities, as shown in figure 6 , have come to IHEP early this month. One of the two $500 \mathrm{MHz} / 250 \mathrm{~kW}$ klystrons and their power supplies have been installed and passed the acceptance test. The vertical cold test of the \#1 superconducting insertion magnet has been finished at BNL, and will be assembled into the cryostat soon. Figure 7 is the superconducting insertion magnet coil \#1 in the wiring stand. The vertical cold test of the \#2 superconducting insertion magnet has been scheduled in June. 
The construction of the cryogenic system is on the critical path. Two $500 \mathrm{~W}$ repudiators were installed and being tested. The bidding for the cryogenics devices, such as valve boxes, cold boxes and transfer lines were carried out, and will be delivered for system integration by August 2005.
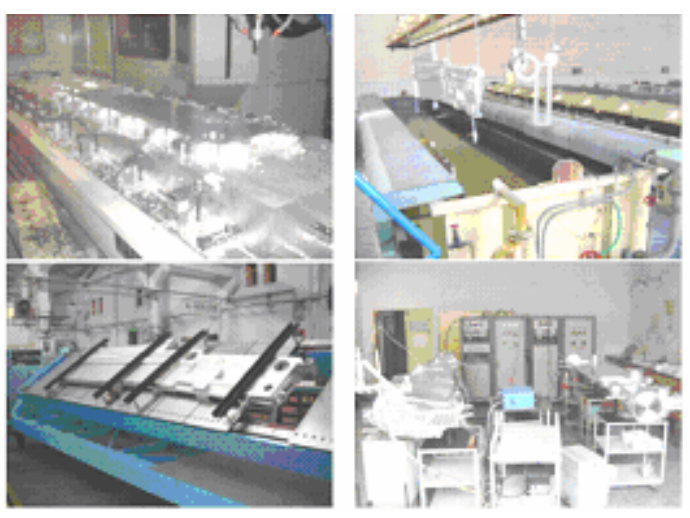

Figure 5: The pictures of the antechamber.

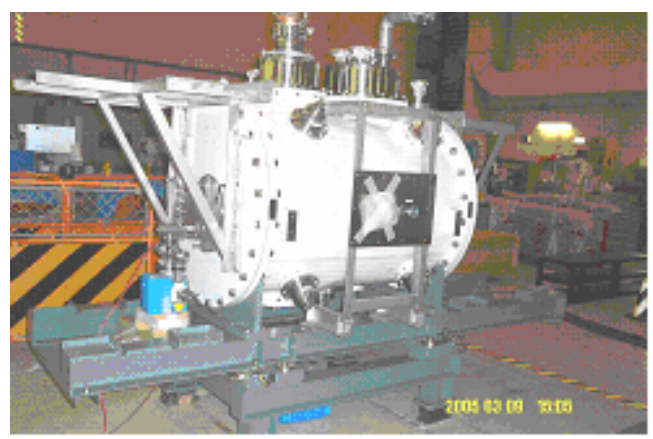

Figure 6: The picture of superconducting cavity.

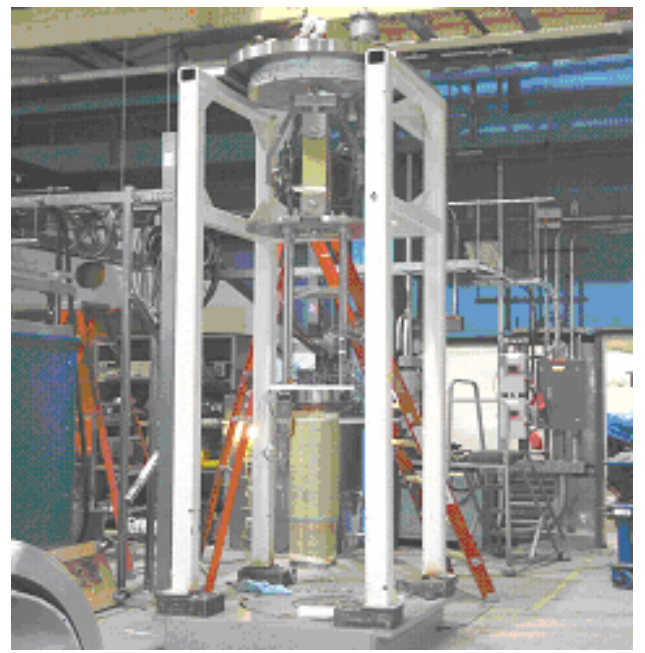

Figure 7: The superconducting insertion magnet coil \#1 in the wiring stand.

\section{SUMMARY}

- The BEPC has been well operated with many exciting HEP and SR results for 15 years since it was put into operation in 1989.

- The BEPCII is designed with a double-ring structure and its design luminosity is two orders of magnitude higher than the present BEPC.

- Some key technologies are being developed in order to achieve the scientific goals of the BEPCII.

- So far, each subsystem seems going smoothly, and can be completed on schedule. The attention should be paid to the superconducting system and cryogenic system integrating, testing and commissioning.

\section{REFERENCES}

[1] S.X.Fang and S.Y.Chen, The Beijing ElectronPositron Collider, Proc. 14-th Int. Conf. on High Energy Acc., 1989, p51.

[2] BEPCII Group, BEPCII Design Report, IHEPProceedings, Aug. 2001.

[3] C.Zhang, et al, Performance of the BEPC and Progress of the BEPCII, Proc.of APAC04, March 22 26, 2004, Gyeongju, Korea.

[4] L.M Chen, et al, Workshop on e+ e- in the 1-2 GeV Energy Range: Physics and Accelerator Prospects, Sept. 2003, Alghero (SS), Italy.

[5] J.Q. Wang et al., Proceedings of EPAC04.

[6] G.X.Pei, et al, Progress of the BEPCII Linac Upgrade, these Proceedings. 